\title{
COUPLING CURRENTS IN RUTHERFORD CABLES UNDER TIME VARYING CONDITIONS
}

\author{
A.P. Verweij and H.H.J ten Kate \\ University of Twente, Applied Superconductivity Centre \\ PO Box 217, NL 7500 AE Enschede, The Netherlands
}

\begin{abstract}
A new network model is presented to simulate fully transposed Rutherford cables under time varying conditions. The intrinsic properties of the cable and the external applied conditions can be changed spatially. Several statistical distributions of the contact resistances are built in to investigate local differences in the coupling loss and in the eddy currents. The average loss is rather independent of a resistance distribution but locally both the loss and the eddy currents can increase significantly. The self field distribution of the cable is included resulting in a saturation of the strands which depends on the relative direction between the magnetic field, the field sweep rate and the transport current. Furthermore, mutual inductances between strands are introduced which enables the use of the model for nonstationary problems (e.g.: the response of the coupling currents to changes in the transport current and/or changes in field sweep rate, including high frequency calculations). Time constants can be calculated for both the coupling currents in the strands as for the local and global dissipation.
\end{abstract}

\section{INTRODUCTION}

Rutherford cables are in common use in magnet windings in which they are exposed to time varying conditions (e.g. the begin and the end of a field sweep in accelerator magnets or in tokamaks). However, existing models ([1],[2],[3]) can only handle stationary cases. The model described here has several important improvements compared to other ones, namely:

- The mutual inductances between strands are implemented so that the model can also handle nonstationary problems (nonconstant transport current or field sweep $\mathrm{dB} / \mathrm{dt}$, response to a $\mathrm{dB} / \mathrm{dt}$ step, high frequency calculations).

- The self field distribution of the cable is included. For small transport currents the self field is not very important but for high transport currents the self field influences the way in which the strands become saturated.

- Statistical distributions of the contact resistances are built in to simulate a cable more realistically and to investigate local differences in the eddy currents and dissipation.

Manuscript received August 24, 1992

\section{U-I RELATION OF A STRAND}

The voltage along (a part of) a strand consists of two terms: an inductive part $U_{s, \text { ind }}$ and a resistive part $\mathrm{U}_{\mathrm{s}, \mathrm{res}}$. The inductive part $\mathrm{U}_{\mathrm{s} \text {,ind }}$ is determined by:

$$
\mathrm{U}_{\mathrm{s}, \text { ind }}^{\mathrm{i}}=\Sigma \mathrm{M}^{\mathrm{i}, \mathrm{j}} \mathrm{dI}^{\mathrm{j}} / \mathrm{dt} \text {, }
$$

where $\mathrm{j}$ has to be taken over all the strand elements in the cable $(\mathrm{j}=\mathrm{i}$ included). The mutual inductances are calculated with formulas given by Grover ([4]).

To determine $U_{s, r e s}$ it is necessary to know the maximum superconducting strand current $I_{\max }$. This relation is given by Hartmann ([5]) stating that $I_{\max }$ not only depends on $B$ but also on $\mathrm{dB} / \mathrm{dt}$ due to the additional screening currents in the filaments:

$$
I_{\max }(\mathbf{B}, \dot{\mathbf{B}})=I_{c}(\mathbf{B})\left[1-\frac{\mathrm{r}_{s}|\dot{\mathbf{B}}|}{\rho_{\perp}(\eta, \bar{B}) \eta J_{c}}\left(\frac{L_{p}, f}{2 \pi r_{s}}\right)^{2}\right],
$$

where $L_{p, f}$ and $r_{s}$ are the filament twist pitch and the strand radius respectively. In this relation $B$ and $\mathrm{dB} / \mathrm{dt}$ consist of the (change of the) applied field $\mathbf{B}_{\mathrm{A}}$ and of the (change of the) self field $\mathbf{B}_{\mathrm{sf}}$. The transverse resistivity of the copper matrix depends on the magnetic field and on the superconducting filling factor $\eta$, so:

$$
\rho_{\perp}(\eta, \mathbf{B})=\rho_{0}(1+\alpha|\mathbf{B}|)(1+\eta) /(1-\eta)
$$

In the case the strand is just saturated the voltage across a part of a strand with length $l_{\mathrm{s}}$ is given by (see [5]):

$$
\mathrm{U}_{\max }=\frac{8 \mathrm{r}_{\mathrm{f}} l_{\mathrm{s}}|\mathbf{\mathbf { B }}|}{3 \pi}
$$

where $\mathbf{r}_{\mathrm{f}}$ is the filament radius.

So, two cases can be distinguished:

1) A strand element is unsaturated $\left(\left|I_{s}\right|<I_{\max }\right)$ :

$$
\begin{aligned}
& U_{s, \text { res }}=I_{s} /\left(I_{\max } / U_{\max }+1 / R_{c o p}\right), \\
& \text { with: } R_{c o p}=\rho_{\| \mid} l_{s} /\left(\pi r_{s}^{2}\right), \\
& \text { and: } \rho_{\|}(\eta, B)=\rho_{0}(1+\alpha|B|)(1+\eta) \text {. }
\end{aligned}
$$

2) A strand element is saturated $\left(\left|I_{s}\right|>I_{\max }\right)$. In this case the surplus of the current $\left(\left|I_{s}-I_{\max }\right|\right)$ flows into the copper matrix which causes a voltage:

$$
\mathrm{U}_{\mathrm{s}, \mathrm{res}}=\mathrm{R}_{\mathrm{cop}}\left(\mathrm{I}_{\mathrm{s}}-\mathrm{I}_{\max }\right) \text {. }
$$




\section{DESCRIPTION OF THE MODEL}

The Rutherford cable is modeled by a three dimensional network of nodes interconnected by strands and by resistances (see Fig. 1).

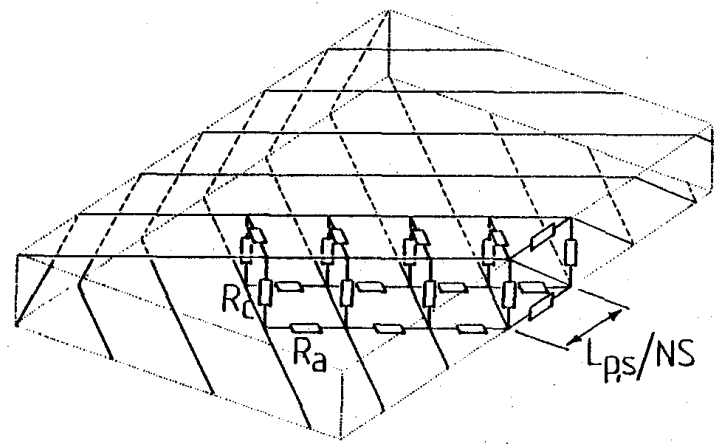

Fig. 1: Network model of a Rutherford cable.

At both edges the strands follow an askew path from one to the other layer. Also the keystone of the cable is implemented. The resistances $R_{c}$ and $R_{a}$ represent the resistances between crossing and adjacent strands respectively. The cable is subdivided in calculation bands with a length $L_{p, s} / N S$, where $L_{p, s}$ and NS are the strand twist pitch and the number of strands respectively. Each band, consisting of (NS-1) calculation cells, has (5NS-3) unknown, namely (2NS-2) strand currents $I_{s}, 2 N S$ currents between adjacent strands $I_{a}$ and (NS-1) currents between crossing strands $I_{c}$.

Using Kirchhoff's formula's the (5NS-3) equations needed to solve one band can be set up. These consist of (2NS-2) equations in the nodes:

$$
\Sigma \mathrm{I}_{\mathrm{a}}+\Sigma \mathrm{I}_{\mathrm{c}}+\Sigma \mathrm{I}_{\mathrm{B}}=0,
$$

(3NS-2) equations for a circuit:

$$
\Sigma\left(I_{a} \cdot R_{a}\right)+\Sigma\left(I_{c} \cdot R_{c}\right)+\sum U_{s, r e s}+\sum U_{s, \text { ind }}=d B_{\perp} / d t \cdot A,
$$

(where $\mathrm{dB}_{\perp} / \mathrm{dt}$ is the component of $\mathrm{dB} / \mathrm{dt}$ perpendicular to $A$ and $A$ is the enclosed surface of the circuit), and one equation:

$$
\text { - } \quad \Sigma I_{a}+\Sigma I_{8}=I_{t r} \text {, }
$$

stating that all currents flowing through the cross section of the cable add up to the transport current.

The equations for band $n$ contain not only currents of band $n$ but also currents of band (n-1) and band $(n+1)$. This implies that for the first and the last band certain boundary conditions are required. Three different cases can be simulated:

- All variables are constant in the z (longitudinal) direction of the cable. In this case only one band (with slightly different equations) has to be calculated to simulate an infinite cable.

- The variables are not constant in the $\mathrm{z}$ direction of the cable. In this case the whole cable has to be calculated resulting in NS(5NS-3) equations for one twist pitch of cable. A small error is made by setting the currents in the first 2 bands equal to each other, and the same for the last two bands.

- Only a small part of the cable is taken simulating a short sample (loss) measurement. Obviously the equations for the first and the last band are slightly different in this case.

For those cases where $\mathrm{dB} / \mathrm{dt}$ is constant for at least a time which is far larger than all the time constants of the system, one can set $U_{\text {sind }}=0$ in eq. (10). However for any nonstationary problem $\left(\mathrm{d}^{2} \mathbf{B} / \mathrm{dt}^{2} \neq 0, \quad \mathrm{dI}_{\mathrm{t} t} / \mathrm{dt} \neq 0\right)$ also the self and mutual inductances of the strands become important. In this case eq. (10) is modeled as follows:

$$
\begin{aligned}
& \Sigma\left(I_{\mathrm{a}} \cdot R_{\mathrm{a}}\right)+\Sigma\left(\mathrm{I}_{\mathrm{c}} \cdot \mathrm{R}_{\mathrm{c}}\right)+\Sigma\left(\mathrm{U}_{\mathrm{s}, \mathrm{res}}^{\mathrm{i}}+\Sigma\left(\mathrm{M}^{\mathrm{i}, \mathrm{j}} \mathrm{I}_{\mathrm{n}}{ }^{\mathrm{j}} / \Delta \mathrm{t}\right)\right]= \\
& \mathrm{d} \mathbf{B}_{\perp} / \mathrm{dt} \cdot \mathrm{A}+\Sigma \Sigma\left(\mathrm{M}_{\mathrm{n}-1}^{\mathrm{j}}, \mathrm{j} / \Delta \mathrm{t}\right),
\end{aligned}
$$

with: $\Delta \mathrm{t}=\mathrm{t}_{\mathrm{n}}-\mathrm{t}_{\mathrm{n}-1}$.

Each case is solved iteratively. For cases without saturation of the strands an accurate solution is already obtained in the first convergence step. Due to the strong nonlinearity of $\mathbf{R}_{\mathbf{s}}$ more steps are normally needed when strands become saturated. An even faster convergence is obtained by stating that a strand can not go from positive saturation to negative saturation (or opposite) and by introducing a convergence constant $\mathrm{c:}$

$$
R_{s}=\left((1-c) \cdot R_{s}^{\text {old }}+c \cdot R_{s}\right)
$$

\section{RESULTS}

A survey of the results as calculated for various cases is given in Table 1. For each case the

Table 1: Survey of results for various cases.

\begin{tabular}{lllll}
\hline Case & $\begin{array}{l}\mathrm{P}_{\mathrm{a}} \\
\mathrm{mW} / \mathrm{m}\end{array}$ & $\begin{array}{l}\mathrm{P}_{\mathrm{c}} \\
\mathrm{mW} / \mathrm{m}\end{array}$ & $\begin{array}{l}\mathrm{I}_{\text {e,max }} \\
\mathrm{A}\end{array}$ & $\begin{array}{l}\mathrm{P}_{\mathrm{cmax}} \\
\mu \mathrm{W}\end{array}$ \\
\hline $\mathrm{A} 1$ & 2.6 & 33.6 & 8.3 & 24.8 \\
$\mathrm{~A} 2$ & 0.13 & 0 & 0.05 & 0.13 \\
$\mathrm{~A} 3$ & 2.6 & 0.7 & 0.4 & 1.2 \\
$\mathrm{~B} 1$ & 2.6 & 35.4 & 10.0 & 25.9 \\
$\mathrm{~B} 2$ & 2.6 & 52.5 & 11.9 & 82.7 \\
$\mathrm{~B} 3$ & 2.6 & 31.4 & 9.0 & 16.7 \\
$\mathrm{~B} 4$ & 2.6 & 38.3 & 20.8 & 53.6 \\
$\mathrm{~B} 5$ & 2.6 & 36.9 & 28.6 & 41.6 \\
$\mathrm{C} 1$ & 2.9 & 37.1 & 8.9 & 26.5 \\
\hline
\end{tabular}


dissipation in $R_{a}$ and in $R_{c}\left(P_{a}\right.$ and $P_{c}$ respectively), the maximum eddy current in a strand $I_{e, \max }$ and the maximum dissipation in a contact $P_{c, \max }$ are given. The results are briefly discussed in paragraph $V$.

\section{A. 'Standard case'}

First of all the results will be given of the calculation of the currents and powers in the 'standard case', i.e. an infinite cable with equal resistances, no space variation of external conditions, a transport current far below $I_{\max }$ and cable parameters as given in Table 2. A 16 strand standard cable is chosen in stead of the more commonly used 26 or 40 strand cables in order to reduce CPU time.

Table 2 Parameters of the 'standard cable'

\begin{tabular}{ll}
\hline $\mathrm{Nr}$. of strands: & 16 \\
$\mathrm{~dB} / \mathrm{dt}$ & $0.1 \mathrm{~T} / \mathrm{s}$ perp. to the wide side \\
$\mathrm{R}_{\mathrm{a}}$ & $10 \mu \Omega$ \\
$\mathrm{R}_{\mathrm{c}}$ & $10 \mu \Omega$ \\
$\mathrm{L}_{\mathrm{p}}$ & $0.1 \mathrm{~m}$ \\
$\mathrm{Cr}_{\mathrm{g}}$ section & $3.40 / 3.20 \times 12.8 \mathrm{~mm}^{2}$ \\
$\mathrm{~B}_{\mathrm{c}, 0}(4.2 \mathrm{~K})$ & $10.7 \mathrm{~T}$ \\
$\mathrm{~J}_{\mathrm{c}, 0}$ & $8000 \mathrm{~A} / \mathrm{mm}^{2}$ \\
$\eta$ & 0.35 \\
$\mathrm{r}_{\mathrm{f}}$ & $10 \mu \mathrm{m}$ \\
\hline
\end{tabular}

For $\theta=0 \quad(d \mathbf{B} / \mathrm{dt}$ perpendicular to the wide side of the cable) the loss contribution is mainly coming from $R_{c}$ (case A1). Only for $\sin (\theta) » \cos (\theta)(\theta=\pi / 2$ : case A2) or for $R_{a} \ll R_{c}$ or in the case of an insulating strip between the two layers (case A3) the loss contribution in $R_{a}$ becomes relatively large. All the currents flowing through the $R_{c}$ 's from one layer to the other return through the two strands at both edges of the cable. The dissipation in the contacts $P_{c}$ along the wide side of the cable is shown in Fig. 2

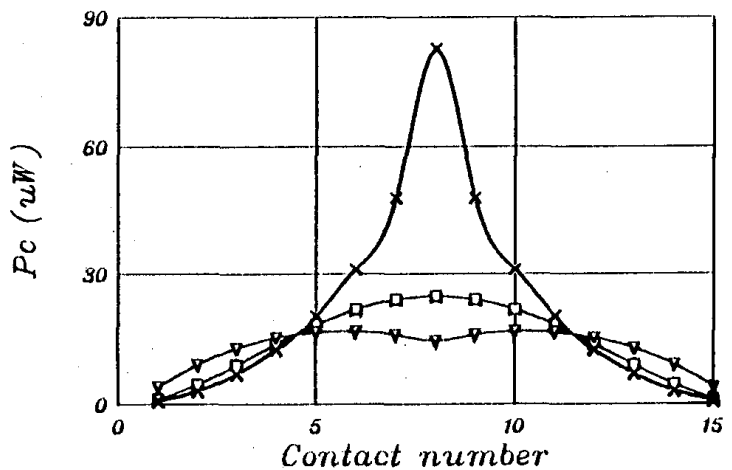

Fig. 2: Dissipation in the contacts along the wide side of the cable for three cases $(\square: A 1, X: B 2$ and $\nabla$ : B3) with different $R_{c}$ distribution.

\section{B. (Statistical) distributions of $R_{\mathrm{c}}$}

Keystoned Rutherford cables are often used in dipole (accelerator) magnets; this keystone will probably result in a gradient of the $R_{c}$ value over the wide side of the cable. Case B1 simulates a linear increase of $R_{c}$ from 5 to $15 \mu \Omega$ over the wide side of the cable. Furthermore to cases are simualated: case $B 2$ with $R_{c}=3 \mu \Omega$ in the middle of the cable and linear increasing to $17 \mu \Omega$ at both edges and case $B 3$ with $R_{c}=3 \mu \Omega$ at the edges and linear increasing to $17 \mu \Omega$ in the middle. In all the cases the average $R_{c}$ is equal to the $R_{c}$ in the 'standard case'. Fig. 2 shows $P_{c}$ along the wide side of the cable for the cases A1, B2 and B3. Case B1 is not included as the distribution is nearly the same (except for a small asymmetry) as for case A1.

It is obvious that the resistances in reality have a certain distribution. First of all a normal distribution $R_{c}=10 \pm 6 \mu \Omega$ is taken over $1 \mathrm{~m}$ of cable (case B4). However, neighboring contact resistances are probably not completely independent. For this reason for case B5 the resistances are given a random value with the restriction that neighboring resistances can only have slightly different values. In this way a cable is simulated having areas (in the order of a few $\mathrm{cm}^{2}$ ) with large and areas with small $R_{\mathrm{c}}$.

\section{Gradient in $d B / d t$}

During charging or discharging of acceleratormagnets, $\mathrm{dB} / \mathrm{dt}$ has a gradient over the wide side of the cable. This case is simulated, taking a linear increase of $\mathrm{dB} / \mathrm{dt}$ from 0.05 to $0.15 \mathrm{~T} / \mathrm{s}$ (case $\mathrm{C} 1$ ). The average $\mathrm{dB} / \mathrm{dt}$ is equal to the $\mathrm{dB} / \mathrm{dt}$ in the 'standard case'.

\section{Self field}

The self field is important in the calculation of $I_{\max }$ (eq. (2)) and it determines the way in which the strands become saturated. In Fig. 3 two cases are shown: case D1 with $\mathbf{B}>0$ and $\mathrm{dB} / \mathrm{d} t<0$ and case D2 with $\mathbf{B}>0$ and $\mathrm{dB} / \mathrm{d}>0$.

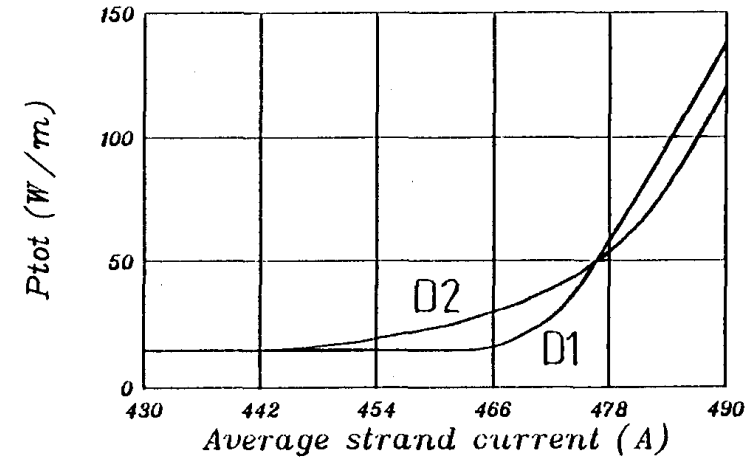

Fig. 3: Total dissipation for the cases D1 and D2 as a function of the average strand current. 


\section{E. Nonstationary conditions}

A point of great interest is the time behavior of the eddy currents and the dissipation due to a change in the transport current and/or a change in $\mathrm{dB} / \mathrm{dt}$ $\left(\mathrm{d}^{2} \mathrm{~B} / \mathrm{dt}^{2} \neq 0\right)$. As an example the response is calculated due to a $\mathrm{dB} / \mathrm{dt}$ step from 0 to $0.1 \mathrm{~T} / \mathrm{s}$ (with simultaneously a $\mathrm{dI}_{\mathrm{tr}} / \mathrm{dt}$ step from 0 to $160 \mathrm{~A} / \mathrm{s}$. Two more cases with $\mathrm{L}_{\mathrm{p}, \mathrm{s}}$ of $0.12 \mathrm{~m}$ (case E1) and with NS of 20 (case E2) are also shown.

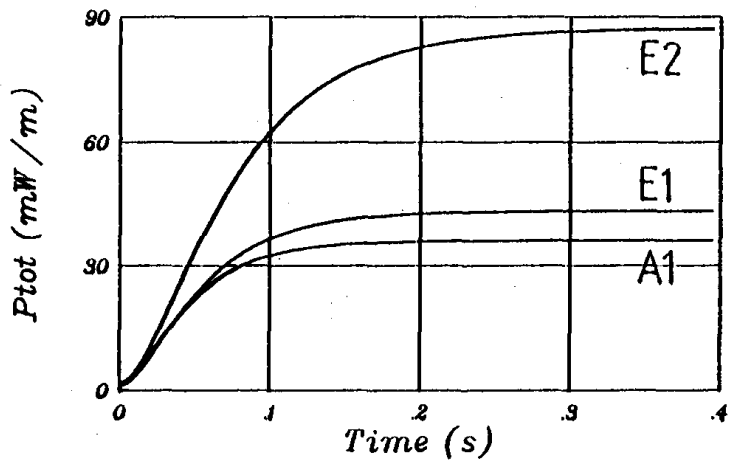

Fig. 4: Response of the total dissipation to a step increase in $\mathrm{dB} / \mathrm{dt}$ (and simultaneously a step increase in $\mathrm{dI}_{\mathrm{tr}} / \mathrm{dt}$ ) for three cases (A1: NS=16 and $\mathrm{L}_{\mathrm{p}, \mathrm{s}}=0.1, \mathrm{E} 1: \mathrm{NS}=16$ and $\mathrm{L}_{\mathrm{p}, \mathrm{s}}=0.12, \mathrm{E} 2: \mathrm{NS}=20$ and $\mathrm{L}_{\mathrm{p}, \mathrm{s}}=0.1$ ).

\section{F. Short sample loss}

For experimental loss measurements usually a short sample is taken. For this reason it is important to investigate whether a short sample is representing a long cable well. In Fig. 5 the result is shown of the average coupling loss versus the length of the short sample for $\theta=0$ and $\theta=\pi / 2$.

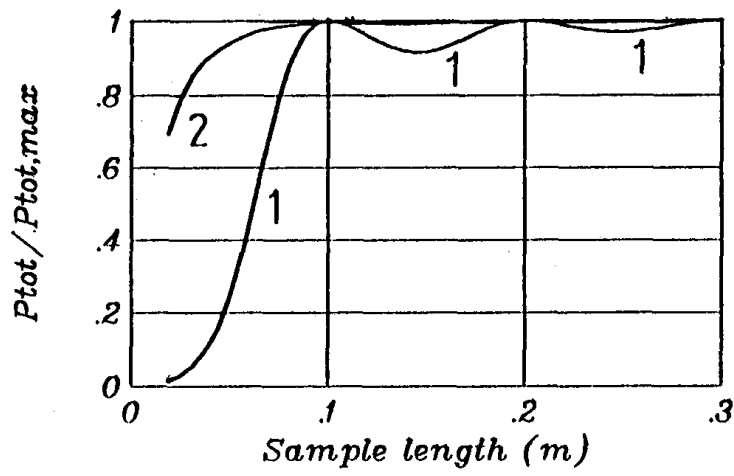

Fig. 5: Scaled coupling loss of a short sample as a function of the sample length for $\mathrm{dB} / \mathrm{dt}$ perpendicular to the wide side of the cable (1) and for $\mathrm{dB} / \mathrm{dt}$ parallel to the wide side of the cable (2). The twist pitch of the strands is $0.1 \mathrm{~m}$.

\section{DISCUSSION AND CONCLUSIONS}

A new network model is developed to simulate keystoned Rutherford cables under time varying conditions. The model is especially very powerful in handling nonstationary cases and situations in which the strands are (partially) saturated. Contact resistances, local critical currents, the field and the field sweep rate can be changed spatially.

Several simulations are performed for a magnetic field sweep perpendicular to the wide side of the cable. Important conclusions are obtained:

- The average dissipation depends especially on the resistances between crossing strands in the middle of the cross section of the cable and on the field sweep rate in the middle of the cable.

- The average dissipation depends slightly on a random distribution of the contact resistances. However locally the loss and the eddy currents can increase significantly. As neighboring contact resistances are probably not independent the dissipation can be high over several $\mathrm{cm}$ and can cause premature quenches if the cooling of the cable is not sufficient. These local differences in the dissipation can not be predicted by a macroscopic measurement of the losses.

- To perform loss measurements on short samples one has to take a sample of at least one twist pitch to relate the losses to a long cable.

- The self field distribution of a cable causes a saturation of the strands which depends on the relative direction between the magnetic field, the field sweep rate and the transport current.

- Mutual inductances between strands are introduced which enables the use of the model for nonstationary problems. High frequency and response cases can be simulated easily. Time constants can be calculated for both the coupling currents in the strands as for the local and global dissipation.

\section{REFERENCES}

[1] G.H. Morgan, Eddy currents in flat metal-filled superconducting braids, J. Appl. Phys., vol. 44, 1973, pp. 3319-3322.

[2] E.M.J. Niessen, D. ter Avest and L.J.M. van de Klundert, Application of the network method to superconducting cables in LTEC90, Southampton, UK, July 1990, pp. 05.4.

[3] V.E. Sytnikov et al., Transport and induced currents distribution in superconducting transposed cables, Adv. Cryog. Eng., vol. 38, pp. 553-558.

[4], F.W. Grover, Inductance calculations, New York: Dover publ., 1962, pp. 17-58.

[5] Hartmann R.A., A contribution to the understanding of AC losses in composite superconductors, PhD thesis, University of Twente, Enschede, The Netherlands, 1989. 